\title{
Sternocleidomastoid muscle can have multiple attachments
}

\author{
Satheesha B. Nayak ${ }^{1}$ (i) $\cdot$ K. V. Soumya ${ }^{2}$ \\ Received: 5 December 2019 / Accepted: 10 January 2020 / Published online: 27 January 2020 \\ ○) Springer-Verlag France SAS, part of Springer Nature 2020
}

\section{Dear Editor,}

We read the article entitled "Variation of the sternocleidomastoid muscle: a case report of three heads and an accessory head" [2] published your esteemed journal. The authors of the article have reported a muscle with three heads of origin and an accessory head. The dissection and photography by the authors is excellent. The variations have been described neatly. According to the authors, the sternocleidomastoid muscle had a sternal head and two clavicular heads. The sternal head gave an accessory belly which joined the lateral clavicular head. What is the basis to call that small fasciculus of sternal head as a separate head when it did not take origin from a bone? What is the basis to say that it merged with the lateral clavicular head? Eventually, all heads of sternocleidomastoid muscle join to form a single belly before its insertion right?

It was better if the authors described the muscle to have three heads of origin (one from the sternum and two from the clavicle). The clavicular heads can be called "medial" and "lateral" clavicular heads. Instead of using the word "additional belly" or "head", it is better to describe it as "an additional fasciculus got separated from the sternal head and merged with the lateral clavicular head".

Sternocleidomastoid muscle is known to show variations in its origin. Up to five fleshy bellies and thirteen heads of origin of this muscle have been already reported [3]. Sternocleidomastoid muscle also shows variations in its insertion.
A recent article reports six heads of its insertion along the superior nuchal line [1].

We opine that sternocleidomastoid variations become functionally, clinically, or surgically important only when the lower attachment or upper attachment shows variations. If there is any minor variation in the middle of the muscle, it might not affect the individual's lifestyle or any clinical procedure significantly.

Author contributions SBN drafted the manuscript. KVS did the review of literature and proof reading of the manuscript.

\section{References}

1. Dupont G, Iwanaga J, Altafulla JJ, Lachkar S, Oskouian RJ, Tubbs RS (2018) Bilateral sternocleidomastoid variant with six distinct insertions along the superior nuchal line. Anat Cell Biol 51(4):305-308

2. Heo YR, Kim JW, Lee JH (2019) Variation of the sternocleidomastoid muscle: a case report of three heads and an accessory head. Surg Radiol Anat. https://doi.org/10.1007/s00276-01902388-4

3. Surendran S, Nayak SB, Reghunathan D, Nelluri VM (2016) Sternocleiodomastoid muscle with five fleshy bellies and thirteen heads of origin. Online J Health Allied Sci 15(3):11

Publisher's Note Springer Nature remains neutral with regard to jurisdictional claims in published maps and institutional affiliations.

Satheesha B. Nayak

nayaksathish@gmail.com

1 Melaka Manipal Medical College (Manipal Campus), Manipal Academy of Higher Education, Madhav Nagar, Manipal, Karnataka 576104, India

2 Department of Mathematics, Manipal Institute of Technology, Manipal Academy of Higher Education, Madhav Nagar, Manipal, Karnataka, India 